Revue

Revue de l'histoire des religions

de Ihistoire

des religions

$3 \mid 2006$

Varia

\title{
Des Géants à Dionysos. Mélanges de mythologie et de poésie grecques offerts à Francis Vian
}

édités par D. Accorinti et P. Chuvin, Alessandria, Edizioni dell'Orso, 2003

\section{Alain Ballabriga}

\section{(2) OpenEdition}

Journals

Édition électronique

URL : http://journals.openedition.org/rhr/5174

DOI : $10.4000 /$ rhr.5174

ISSN : 2105-2573

Éditeur

Armand Colin

Édition imprimée

Date de publication : 1 septembre 2006

Pagination : 345-347

ISBN : 2200-92105-5

ISSN : 0035-1423

Référence électronique

Alain Ballabriga, « Des Géants à Dionysos. Mélanges de mythologie et de poésie grecques offerts à

Francis Vian », Revue de l'histoire des religions [En ligne], 3 | 2006, mis en ligne le 22 janvier 2010 ,

consulté le 22 septembre 2020. URL : http://journals.openedition.org/rhr/5174 ; DOI : https://doi.org/ $10.4000 /$ rhr.5174

Ce document a été généré automatiquement le 22 septembre 2020.

Tous droits réservés 


\title{
Des Géants à Dionysos. Mélanges de mythologie et de poésie grecques offerts à Francis Vian
}

\author{
édités par D. Accorinti et P. Chuvin, Alessandria, Edizioni dell'Orso, 2003
}

\section{Alain Ballabriga}

\section{RÉFÉRENCE}

Des Géants à Dionysos. Mélanges de mythologie et de poésie grecques offerts à Francis Vian, édités par D. Accorinti et P. Chuvin, Alessandria, Edizioni dell'Orso, 2003, $24 \mathrm{~cm}$, XL-640 p. (« Hellenica » 10), $75 €$

1 Ces Mélanges en l'honneur de l'helléniste Francis Vian offrent 48 contributions classées en 4 parties: 1. Études mythologiques, 2. Poésie archaïque et classique, 3. Poésie hellénistique et romaine, 4. Études nonniennes et Antiquité tardive. Nombre de ces études, tout à fait pertinentes eu égard aux travaux du dédicataire, sont de nature à intéresser les littéraires plutôt que les historiens des religions. Aussi a-t-il semblé opportun de sélectionner les études qui semblent plus propres à cette dernière discipline, bien que le partage, en matière de poésie grecque, entre le littéraire et le religieux n'aille pas sans quelque arbitraire.

Dans la première partie, on peut ainsi d'abord relever une étude qui, s'opposant à une tendance hypercritique, rappelle les données permettant de penser que le mythe de Dionysos et des Titans faisait sans doute partie de l'orphisme ancien des temps classiques (Alberto Bernabé, p. 25-39).

Deux contributions touchent au problème de l'héritage indo-européen en Grèce ancienne. La première analyse des éléments de la mythologie guerrière des Grecs, comparables à ceux qu'offrent les guerriers-fauves (berserkir) de la mythologie germanique (Pierre Sauzeau, p. 95-108). La deuxième montre qu'une même pensée régit le discours sur les reines et les rois de Sparte en opposant deux formes 
complémentaires de la souveraineté, tournées l'une vers la guerre, l'autre vers l'activité civile (Bernard Sergent, p. 109-134).

Dans la dernière étude de cette partie, les auteurs montrent, contre Bowersock, que les fêtes alexandrines de l'Éternité (Aiôn), documentées par Épiphane (315-403), évêque de Salamine de Chypre, dans son Panarion, "Boîte à remèdes " (375-378), ne sont pas influencées par le christianisme et proposent une interprétation gréco-égyptienne de ces fêtes (Jean Yoyotte et Pierre Chuvin, p. 135-145).

5 Une étude de la deuxième partie repose le problème de la datation et partant de l'interprétation de certaines pièces des Hymnes Homériques.

6 Transmise dans un corpus hymnique comprenant aussi les Hymnes de Callimaque, les Argonautiques Orphiques, les Hymnes Orphiques, les Hymnes du néo-platonicien Proclos ( $\mathrm{V}^{\mathrm{e}}$ s. ap. J.-C.), la collection des hymnes dits «homériques », à côté de pièces remontant pour l'essentiel aux périodes archaïques et classiques, présente quelques poèmes tardifs comme l'Hymne à Arès. L'auteur propose encore de voir dans l'Hymne à Hestia une pièce tardive influencée par la théologie néo-platonicienne (Thomas Gelzer, p. 155-162).

7 Essentiellement consacrée à Apollonios de Rhodes et à la poétique hellénistique (imitation, intertextualité), la troisième partie offre trois études d'une portée différente. L'étape des Argonautes sur l'île d'Anaphè dans les Argonautiques d'Apollonios, outre le dialogue intertextuel avec les Origines (Aitia) de Callimaque, renvoie à un culte réel: les lazzis que les femmes lancent aux hommes lors d'un banquet prenaient place dans une fête à Apollon Asgelatas, hellénisé en Aiglètès et que l'on peut aussi rapprocher de gelôs "rire" (Pierre Chuvin, p. 215-221). Deux contributions concernent les rapports entre le judaïsme et l'hellénisme: les témoignages des Anciens, depuis Philon d'Alexandrie, relatifs à une poésie hébraïque pensée en termes de métrique grecque, renvoient fondamentalement à l'idée d'une poésie biblique comparable, voire supérieure, à celle des Grecs et des Romains (Antoine Hilhorst, pp. 305-329); dans les Oracles Sibyllins, le thème des Géants et des Titans présente une combinaison d'éléments hésiodiques et bibliques (Jane Lightfoot, pp. 393-401).

8 Selon la même optique, dans les riches études nonniennes de la quatrième partie, on retiendra ce qui touche au problème fondamental, passionnant et mal connu des " classicistes », posé par la coexistence des cultures païenne et judéo-chrétienne dans l'Antiquité tardive et à Byzance.

9 Ainsi l'étude des poésies de l'Égyptien Dioscore d'Aphroditô (ca. 520-585) ne peut se restreindre à celle de l'imitation littéraire des Dionysiaques et de la Paraphrase de l'Évangile selon saint Jean de Nonnos de Panopolis ( $\mathrm{V}^{\mathrm{e}} \mathrm{s}$.). Il faut aussi tenir compte de la pensée et de la pratique eucharistique dans une Égypte de l'Antiquité tardive qui est par ailleurs bilingue (grec, copte). Si la Paraphrase de Nonnos et les poèmes de Dioscore présentent une "heureuse interpénétration du style homérique et de la liturgie égyptienne ", c'est que la liturgie était aussi centrale pour la vie des élites qui devaient à leur éducation (paideia) classique d'occuper le rang qui était le leur (Leslie Mac Coull, p. 489-500).

10 La persistance des formes classiques au siècle de Justinien $\left(\mathrm{VI}^{\mathrm{e}} \mathrm{s}\right.$.) peut encore s'illustrer par la Description de Sainte-Sophie, composée en hexamètres nonniens par Paul le Silentiaire en 562 (Marie-Christine Fayant, pp. 583-592 ; Mary Whitby, pp. 539-606). Au siècle suivant, le passage au dodécasyllabe byzantin s'observe dans le poème Sur le 
rétablissement de la Croix de Georges de Pisidie, diacre de Sainte-Sophie durant le règne d'Héraclius (610-641) (André-Louis Rey, p. 607-620). Néanmoins, on pourra encore composer des poèmes hexamétriques dans le style de la Paraphrase de Nonnos au temps de la Renaissance byzantine du $\mathrm{IX}^{\mathrm{e}} \mathrm{s}$. : c'est le cas en particulier de la Résurrection de Lazare de Comètas (Anthologie Palatine, $\mathrm{xv}, 40$ ). De telles pièces témoignent du «souci chez les lettrés de la Renaissance byzantine de récupérer la grande tradition classique à travers les auteurs tardo-antiques dont la médiation était indispensable » (Francesco Tissoni, p. 621-635).

11 Outre ce groupe d'études sur la signification de la réception de Nonnos $d u \mathrm{VI}^{\mathrm{e}} \mathrm{au} \mathrm{IX}^{\mathrm{e}} \mathrm{s}$., on signalera pour finir deux autres travaux remarquables sur l'histoire culturelle et sociale de l'Antiquité tardive.

Sur la mosaïque recouvrant le sol de la basilique de Qasr el-Lebia (l'antique Théodorias en Cyrénaïque, ainsi nommée par Justinien en l'honneur de son épouse Théodora), la représentation de la fontaine Castalie permet d'intégrer le passé mythique, en particulier de la Cyrénaïque, dans la tradition chrétienne, qui récupère les oracles apolliniens (de Delphes et/ou d'Antioche) et les dépasse dans le cadre de la Révélation (Gianfranco Agosti, pp. 541-564).

13 À la suite de cette analyse iconographique, on peut lire une étude, à la lumière de l'épigraphie et de la psychologie sociale, sur la place des Juifs dans les conflits entre les deux principales factions du cirque, les Verts (Prasinoi) et les Bleus (Benetoi) : en tant que sous-groupe bien défini des Bleus, les Juifs étaient en butte de la part des Verts à des attaques s'expliquant à la fois par des sentiments anti-judaïques et par un comportement violent relevant du pur et simple "hooliganisme » qui accompagne encore de nos jours certaines manifestations sportives (Pieter w. van der Horst, p. 565-571).

\section{AUTEUR}

\section{ALAIN BALLABRIGA}

Centre national de la Recherche scientifique, Toulouse 